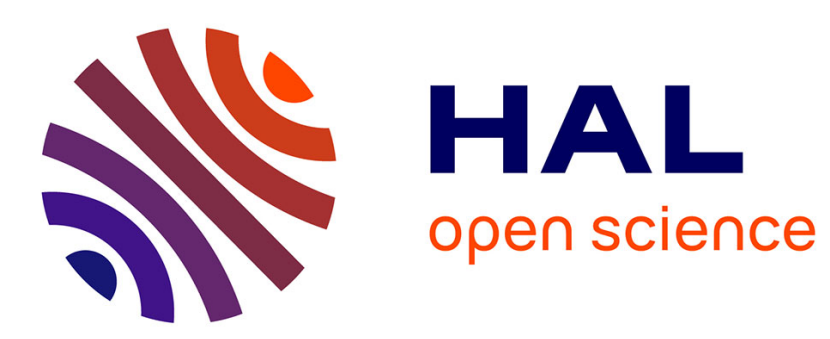

\title{
Réinventer l'entreprise: la gestion collégiale des inconnus communs non appropriables
}

\author{
Pascal Le Masson, Benoit Weil
}

\section{To cite this version:}

Pascal Le Masson, Benoit Weil. Réinventer l'entreprise: la gestion collégiale des inconnus communs non appropriables. L'entreprise, point aveugle du savoir, 2014. hal-01083252

\section{HAL Id: hal-01083252 \\ https://hal.science/hal-01083252}

Submitted on 17 Nov 2014

HAL is a multi-disciplinary open access archive for the deposit and dissemination of scientific research documents, whether they are published or not. The documents may come from teaching and research institutions in France or abroad, or from public or private research centers.
L'archive ouverte pluridisciplinaire HAL, est destinée au dépôt et à la diffusion de documents scientifiques de niveau recherche, publiés ou non, émanant des établissements d'enseignement et de recherche français ou étrangers, des laboratoires publics ou privés. 


\title{
Réinventer l'entreprise : la gestion collégiale des inconnus communs non appropriables
}

\author{
Pascal Le Masson, Benoit Weil
}

Plus organisées que le marché, plus innovantes que les institutions, les entreprises sont souvent apparues comme des espaces privilégiés pour inventer de nouvelles formes d'action collective et pour explorer de nouveaux inconnus. Pourtant ces rationalisations elles-mêmes se sont souvent travaillées dans des collectifs dépassant très largement le cadre de l'entreprise comme en témoignent les mouvements célèbres comme la Lunar Society, ou le mouvement pour la recherche industrielle... Dans les interstices des écosystèmes, nous proposons d'appeler "collèges de l'inconnu " ces collectifs qui se forment là où n'est pas encore l'entreprise. Ces collèges aux configurations variées explorent les marges de l'inconnu non encore imaginable ou pensable et s'aventurent ainsi dans des espaces encore hors d'atteinte pour les entreprises. Ce faisant, ils dessinent aussi, en négatif, les impossibles d'une entreprise tributaire des contraintes d'appropriation et invitent à renouveler les perspectives sur les milieux nécessaires pour inventer les formes nouvelles de l'action collective.

\section{Les entreprises, l'inconnu et la conception}

Avec l'époque moderne, l'activité humaine s'est engagée résolument dans l'exploration de l'inconnu en s'efforçant de repousser les limites de l'inconnu qu'elle était en mesure de penser et d'explorer. Les théâtres de machines de la Renaissance (VérIN, 1993) avaient pour ambition de faire découvrir à un public cultivé les incroyables possibilités des machines inventées par les ingénieurs. Les savants, grâce à de nouveaux instruments comme le télescope ou le microscope se sont ensuite attachés à faire une science de l'invisible. Ils se sont intéressés à des phénomènes qui n'étaient jusque-là pas directement accessibles aux sens, autour du vide, du magnétisme, de l'électricité... Le mouvement 
d'exploration et de déplacement de l'inconnu s'est poursuivi avec la révolution industrielle qui a multiplié objets et machines, matériaux et procédés nouveaux. Ces efforts de conception sont souvent incarnés par des grandes figures d'innovateurs-entrepreneurs comme Watt et Boulton pour la machine à vapeur ou les Stephenson pour le chemin de fer ; mais ils sont également le fait de tout un milieu composé de techniciens (fabricants de montres ou d'instruments, mécaniciens utilisateurs de machines...). Les grandes entreprises naissantes de la deuxième partie du XIX ${ }^{e}$ siècle vont poursuivre ce mouvement en l'amplifiant et en organisant ces activités de conception.

Les multiples tentatives pour définir et décrire l'entreprise mettent pourtant rarement l'accent sur les activités de conception. Nous avions montré lors d'un précédent colloque de Cerisy (Le Masson \& WeIL, 2008) que la domestication de la conception à la fin du $\mathrm{XIX}^{\mathrm{e}}$ siècle avait joué un rôle déterminant dans l'émergence des grandes entreprises. Linvention des grands bureaux d'études a été l'un des moyens d'une rationalisation de grande ampleur permettant à la grande entreprise de s'imposer face aux inventeurs entrepreneurs de la première révolution industrielle. Le but de ces bureaux d'études était d'organiser la conception de produits toujours nouveaux et toujours plus performants s'appuyant sur des moyens industriels qu'ils allaient également contribuer à développer et rationaliser. L'entreprise, par sa capacité à organiser en son sein des apprentissages collectifs, apparaissait comme la matrice privilégiée pour donner naissance à ces formes originales d'action collective. Véritables « bureaucraties génératives » ces bureaux d'études et ces départements de R\&D aux effectifs souvent importants, et mobilisant des ingénieurs et des techniciens très compétents génèrent avec une efficacité remarquable grâce à des règles de conception qu' ils ont également conçues l'incroyable variété des produits toujours plus performants, sophistiqués qui se sont répandus au $\mathrm{XX}^{\mathrm{e}}$ siècle (voitures, avions, machines, appareils domestiques, TV, Hifi...) organisant les secteurs industriels et les marchés autour de dominant design.

Pourtant, au cours des vingt dernières années, ce modèle rencontre ses limites. L'informatique, internet et les smart phones sont devenus des exemples emblématiques de l'apparition de ces nouvelles identités avec leur cortège de nouvelles firmes qui sont en permanence menacées 
d'obsolescence si elles ne réussissent à réinventer en permanence les produits et services qu'elles proposent. Les ruptures dans l'identité des objets (Le Masson et al., 2010) touchent tous les secteurs y compris les plus traditionnels: que l'on songe à l'automobile, l'énergie ou la construction, on constate partout le même impératif de renouvellement des produits et des compétences que les firmes peinent parfois à organiser tant il leur est difficile de penser les nouvelles directions à emprunter. Déplacer à nouveau la frontière de l'inconnu redevient donc un enjeu majeur, particulièrement difficile pour les entreprises. Pour essayer d'en comprendre les raisons, nous devons modéliser cette activité d'exploration de l'inconnu, activité qui est restée absente des théories les plus usuelles de l'entreprise.

\section{L'exploration de l'inconnu: une énigme et une limite pour les théories de l'entreprise?}

La théorie économique classique (Arrow \& Debreu, 1954) définit l'entreprise comme une fonction de production qui transforme des quantités de compétences en des quantités de biens grâce à une capacité de production. Ce modèle (figure 1.1) fait l'hypothèse que la firme optimise une fonction " objectif » connue et dispose de connaissances illimitées.

Les théories évolutionnistes ont critiqué cette hypothèse. Pour elles, les connaissances sont limitées et finies mais peuvent être étendues par apprentissage, d'où l'importance des routines dans le modèle qu'elles proposent. Dans ces modèles, les innovations - qui viennent étendre la liste des biens connus au départ, sont dues à ces apprentissages, mais aussi à des idées exogènes, proposées par les entrepreneurs, et susceptibles de provoquer des chocs plus radicaux.

Avec ces idées, les économistes évolutionnistes (Nelson, 1992; Dosi \& MaLerba, 2006) nous invitent donc à penser qu'il y aurait autre chose que la connaissance. Il faudrait modéliser (figure 1.2) un autre espace, celui de l'imaginaire ou plus précisément de l'inconnu. Mais celui-ci est-il infini et librement accessible comme ils le supposent? Les idées des entrepreneurs proviennent-elles d'une réserve 
sans limite et toujours disponible?

Les travaux des psychologues (WARD, SMITH et al., 1999) nous apprennent que les idées ne sont pas toutes immédiatement accessibles. Nous sommes généralement victimes de biais de fixation qui entravent nos efforts pour être créatifs. Si l'on demande à des individus d'être le plus créatifs possible et de dessiner des animaux imaginaires, ceux-ci auront tous des têtes, des yeux, des membres... Les théories de la conception ont également mis en évidence l'importance de ces biais de raisonnements et s'efforcent de proposer des moyens pour aider aux défixations nécessaires pour étendre les imaginables et désigner de nouveaux inconnus.

\section{Figure I : \\ LES THÉORIES DE L'ENTREPRISE}

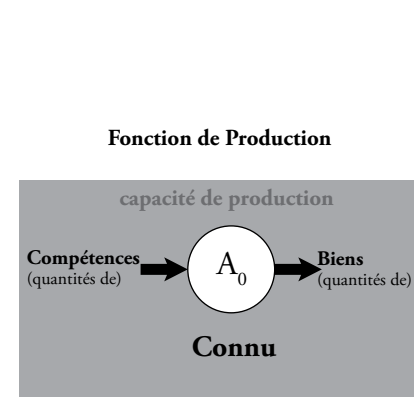

1- Théorie économique classique

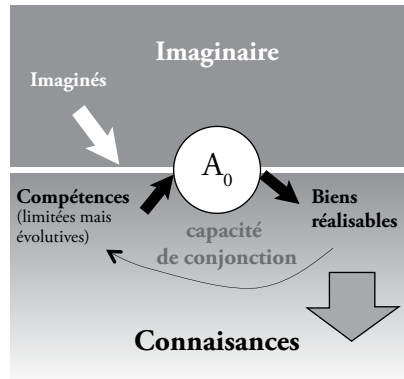

2- Théorie évolutionniste

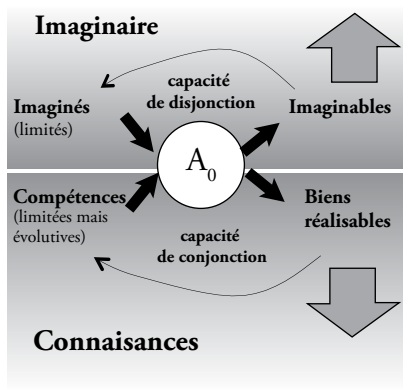

3- Théorie des fonctions de conception

Nous arrivons donc au modèle des fonctions de conception suivant $^{1}$ : le producteur des économistes classiques est devenu un concepteur qui agit dans deux espaces, les connaissances et l'imaginaire (l'inconnu). À partir des connaissances et des inconnus qui lui sont accessibles au départ, il est en état de produire des biens réalisables et de nouvelles connaissances. Mais il peut également, au cours de ce processus, étendre l'espace de l'imaginaire par de nouveaux inconnus

1- Ce modèle, s'appuie sur les fonctions de conception introduites par Le Masson et Hatchuel, a d'abord été proposé par les auteurs dans le cadre du projet RITE de l'ANR (WEIL et al. 2011), il a ensuite été repris notamment par Marine Agogué (2012). 
rendus imaginables, de la même façon qu'il a pu au cours de ce processus étendre par apprentissages les connaissances à sa disposition. L'enjeu devient pour lui d'étendre l'ensemble des biens gérables (ceux pour lesquels il dispose des concepts et des connaissances nécessaires) et l'ensemble des concepts désirables, l'imaginable.

Ce modèle à un seul agent peut facilement être étendu à plusieurs agents en interaction. Sur cette base on peut alors préciser les nouveaux enjeux spécifiques à l'exploration de l'inconnu. Quel est l'inconnu accessible? Pas seulement au sens de l'inconnu réalisable, mais au sens de l'inconnu imaginable. On se focalise généralement sur le premier de sorte qu'on peut souvent être bloqué dans un «imaginable » trop restreint Est-il possible d'étendre l'imaginaire ou plus précisément "l'inconnu imaginable "? Il ne s'agit pas là d'un inconnu «inimaginable " mais bien d'un inconnu encore non imaginé, impensé et impensable jusque-là. L'enjeu est alors de rendre pensables, imaginables des inconnus jusque-là non imaginés.

À ce stade, notre question initiale se précise: existe-t-il des formes d'organisation capables de prendre en charge cet effort d'extension des inconnus imaginables? Si oui, est-ce l'entreprise ou bien d'autres formes? Lesquelles? Et peut-on rationaliser ces processus d'apprentissage d'un nouveau type?

\section{L'entreprise peut-elle organiser l'extension des imaginables?}

Le modèle précédent nous permet de mieux comprendre le rapport de l'entreprise aux activités de conception et à l'exploration de l'inconnu que nous avons évoqué dans le premier paragraphe. Dotée de capacité de conception (bureaux d'études et laboratoires de R\&D), la firme dispose des connaissances qui lui permettent de transformer une partie de l'espace imaginaire-inconnu en produits ou services par une activité de conception. Nous qualifierons d'" inconnu réalisable " cette portion de l'imaginaire atteignable par l'entreprise dans son écosystème actuel. La conception s'accompagne d'apprentissages qui étendent l'espace tant des connaissances que de l'imaginaire. 
Une partie de ce nouvel imaginaire est accessible à l'entreprise à « un effort de conception près ", c'est-à-dire à partir du moment où elle est capable d'enrichir les connaissances et les ressources dont elle dispose dans son écosystème. Nous l'appellerons « inconnu accessible ». L'entreprise peut ainsi étendre l'espace imaginaire sur lequel elle s'installe et qu'elle exploite.

Avec toutefois un risque d'épuisement omniprésent et qui menace toute entreprise lancée dans la compétition contemporaine par l'innovation. Les inconnus réalisables et accessibles par différentes entreprises d'un même secteur risquent fort de se recouper: la compétition porte alors nécessairement sur des produits comparables, ne laissant alors aux entreprises, comme seule variable d'action, que la recherche de gains de productivité ou l'érosion des marges... Face à ce danger, la régénération de l'inconnu, l'extension de l'inconnu imaginable, apparaît comme une alternative fortement souhaitable. Les entreprises doivent pour cela prendre conscience que seule une partie de l'imaginaire leur était accessible, mais qu'il y a toujours une part résiduelle, un inconnu (non accessible) nouveau. L'enjeu pour l'entreprise est alors d'apprendre à déplacer la frontière de l'imaginable accessible.

Historiquement il est possible de repérer de nombreux déplacements de ce type. Le tableau ci-après en donne quelques exemples bien connus et documentés ${ }^{2}$ (voir page suivante).

L'examen de ces exemples, et de nombreux autres analogues, montre que ces déplacements de la frontière de l'imaginable ne sont pas réalisés par une entreprise seule mais qưils mobilisent toujours des tiers, d'autres acteurs et des formes d'organisation qui vont au-delà de son écosystème constitué. Ainsi au milieu du XIx ${ }^{e}$ siècle la question du rattrapage industriel de l'Angleterre est un enjeu et une tension industrielle majeure pour l'Allemagne. Ferdinand Redtenbacher va jouer un rôle majeur dans cette évolution. Il travaille avec la société suisse Escher Wyss concepteur et fabricant de machines réputé avant de proposer et d'enseigner à l'Université de Karlsruhe de nouvelles méthodes de conception paramétriques. Ces recettes de conception reviendront

2- Dans le cadre de cette contribution nous ne pouvons donner toutes les références nécessaires. Sur Redtenbacher et le rattrapage industriel allemand voir König, 1999 ; sur Taylor, voir Nelson, 1980 ; Hatchuel, 1994 ; sur Dudley voir le site de l'ASTM ; sur le Werkbund, voir Schwartz, 1996 ; sur le Bauhaus, voir Droste, 2002 ; sur Hansen et Carl Zeiss, voir Heymann, 2005. 
DÉPLACEMENTS DE LA FRONTIÈRE DE L'IMAGINABLE: LES MUTATIONS DES ACTIVITÉS DE CONCEPTION

\begin{tabular}{|c|c|c|c|c|c|c|}
\hline Période & Tension & Promoteur & Entreprise & $\begin{array}{l}\text { Organisation } \\
\text { impliquée }\end{array}$ & $\begin{array}{l}\text { Inconnu } \\
\text { à explorer }\end{array}$ & $\begin{array}{l}\text { Inconnu } \\
\text { gérable par }\end{array}$ \\
\hline $1840-1850$ & $\begin{array}{l}\text { Rattrapage } \\
\text { industriel }\end{array}$ & Redtenbacher & Escher Wyss & $\begin{array}{l}\text { Université } \\
\text { Karlsruhe }\end{array}$ & Machines adaptées & $\begin{array}{l}\text { Conception } \\
\text { paramétrique } \\
\text { technicien }\end{array}$ \\
\hline $1880-1910$ & $\begin{array}{l}\text { Négociation } \\
\text { salaire } \\
\text { Nouvelles } \\
\text { machines }\end{array}$ & Taylor & Midvale Steel & ASME & Procédés & $\begin{array}{l}\text { Bureau } \\
\text { des Méthodes }\end{array}$ \\
\hline $1875-1900$ & $\begin{array}{l}\text { Rail acier } \\
\text { négociation } \\
\text { client- } \\
\text { fournisseur }\end{array}$ & Dudley & $\begin{array}{l}\text { Pennsylvania } \\
\text { Railroad }\end{array}$ & ASTM & Phénoménologies & $\begin{array}{l}\text { Recherche } \\
\text { industrielle }\end{array}$ \\
\hline $1900-1930$ & $\begin{array}{l}\text { Made in } \\
\text { Germany }\end{array}$ & $\begin{array}{l}\text { Muthesius, } \\
\text { Behrens, } \\
\text { Gropius, } \\
\text { Itten, } \\
\text { Klee }\end{array}$ & AEG & $\begin{array}{l}\text { Werkbund } \\
\text { Bauhaus }\end{array}$ & Faire style & Design \\
\hline 1950 & $\begin{array}{l}\text { Appareils } \\
\text { de précision } \\
\text { (HQ et } \\
\text { expertise) }\end{array}$ & $\begin{array}{l}\text { Hansen, } \\
\text { Bischoff, } \\
\text { Bock }\end{array}$ & $\begin{array}{l}\text { Carl Zeiss } \\
\text { Jena }\end{array}$ & $\begin{array}{l}\text { Hochschule } \\
\text { Ilmenau }\end{array}$ & $\begin{array}{l}\text { Extension } \\
\text { inconnue à } \\
\text { identité connue }\end{array}$ & $\begin{array}{l}\text { Conception } \\
\text { Systématique }\end{array}$ \\
\hline
\end{tabular}

ensuite dans les entreprises. Elles leur permettront de concevoir des familles de machines nouvelles toujours adaptées à leur contexte d'emploi.

On peut schématiser ce mouvement de la façon suivante: une tension désigne un nouvel imaginable inaccessible (en particulier pour les entreprises concernées), le déplacement de la frontière est opéré par un acteur tiers, qui connaît bien la tension et l'entreprise. Cet acteur propose une nouvelle manière de "désigner " et " traiter » l'inconnu encore inaccessible, ce qui permet ensuite à l'entreprise, à partir de ces nouvelles catégories, de déplacer sa frontière de l'imaginable accessible.

Ce même mouvement se retrouve systématisé dans les collèges de l'inconnu comme nous allons le montrer maintenant. 


\section{Comment les collèges de l'inconnu arrivent-ils à augmenter l'inconnu imaginable?}

Dans cette section, nous examinerons deux situations contemporaines où des « collèges de l'inconnu " ont réussi à déplacer la frontière de l'inconnu accessible. Nous dégagerons ensuite quelques caractéristiques de ces collèges de l'inconnu et des formes d'actions collectives qu'ils organisent et qu'ils supportent.

\section{Construire en chanvre ${ }^{3}$}

L'utilisation des fibres de chanvre dans la construction est un bon exemple des blocages de l'innovation orpheline (Agogue, 2012) Pendant plus de quinze ans les nombreuses tentatives sont marquées par un effet de fixation sur la rénovation de constructions traditionnelles. Les expériences restent isolées, très proches les unes des autres et traduisent la difficulté de l'échange des concepts. L'imaginable se limite au traitement des fibres à intégrer dans les torchis et mortiers...

Sur la base de ce constat en 1997, la Chanvrière de l'Aube, coopérative de transformation du chanvre, organise un colloque avec toutes les parties prenantes intéressées qui met en évidence que la promesse de construire en chanvre n'est encore qu'un potentiel, jusque-là assez limité, qui mérite d'être enrichi et qui suppose encore un très important effort de conception. Il ne s'agit pas à ce stade de valider des solutions techniques mais d'étendre le champ d'innovation et d'ouvrir de nouvelles voies.

Une association est créée pour élargir le cercle des acteurs intéressés et prêts à s'investir. L'investissement que l'on attend d'eux n'est pas celui entendu au sens d'une logique de décision ou d'investissement, mais celui de participer à un effort de conception dans une logique d'exploration. L'association permet d'échanger sur les explorations en cours mais également d'ouvrir d'autres voies, de diffuser largement des concepts provocants pour favoriser la défixation, de partager des inconnus (potentiel du champ d'innovation, étendue des connaissances manquantes).

3- Voir Le Masson et al., 2012. 
Rapidement ce collectif est capable de susciter une dynamique et d'ouvrir des voies nouvelles à l'application du chanvre dans le domaine de la construction. Les responsables de l'association s'efforcent d'éviter les phénomènes d'engouement et de bulles spéculatives, en proposant une régénération régulière des concepts stimulants qui permettent de rouvrir en permanence les enjeux de conception et l'entrée dans le jeu de nouveaux acteurs, détenteurs de compétences et d'enjeux différents, intéressés à participer à ces nouvelles explorations.

En peu d'années une nouvelle filière industrielle est née proposant des applications inattendues ayant mobilisés des acteurs très variés et qui au départ ne se sentaient pas forcements concernés.

\section{LITRS ou l'innovation de rupture constante pour suivre la loi de Moore}

La loi de Moore, en promettant tous les dix-huit mois un doublement de la performance des microprocesseurs par miniaturisation des transistors, est étroitement associée à la croissance spectaculaire depuis cinquante ans du secteur des semi-conducteurs. Mais cette loi n'a rien d'une loi naturelle. Elle n'a pu être suivie qu'au prix d'un incessant effort d'innovations radicales renouvelant en permanence les technologies et les compétences nécessaires. On trouve par exemple aujourd'hui presque tous les éléments du tableau de Mendeleïev dans les semi-conducteurs, alors que dans les années 1960 seuls de rares éléments étaient compatibles avec le silicium. Ce rythme et cette intensité d'innovation n'ont été rendus possibles que par un dispositif organisé très original et peu connu : l'ITRS (International Technology Roadmap for Semiconductors).

L'ITRS vise à animer l'écosystème des concepteurs de technologies et des procédés de fabrication des microprocesseurs. Y participent l'ensemble des entreprises concevant ou produisant des semi-conducteurs, leurs fournisseurs et des laboratoires de recherche de rang mondial. Trois réunions par an sont organisées durant lesquelles quinze groupes de travail thématiques échangent sur les concepts techniques à explorer pour soutenir le rythme de la loi de Moore. Ce travail donne lieu à la publication régulière tous les deux ans d'une volumineuse roadmap disponible gratuitement en ligne. 
Le " memorandum of understanding" signé à la création de l'ITRS en 2001 explicite et souligne l'originalité de ses missions: «Sponsoring participants shall cooperate to identifiy generic technology needs for the global semiconductor industry without regard to particular products of individual companies and encourage on an industry-wide basis potential solutions to future technology challenges."

Le but de l'ITRS n'est pas de choisir des solutions techniques, mais d'identifier des problèmes sans solution, les trous dans les technologies disponibles pour être en mesure de suivre la loi de Moore. L'objectif est de mutualiser la connaissance de ces lacunes, de proposer de nouveaux concepts et de focaliser l'effort collectif sur les concepts encore ouverts.

Pour éviter les effets de "lock in " sur des technologies connues et partagées (mais dont l'amélioration de performance ne suffirait pas pour suivre le rythme de la loi de Moore) l'ITRS, fonctionne comme un " collège de l'inconnu " en s'appuyant sur des " unlocking rules» (LE Masson, Weil et al., 2012). Ainsi la loi de Moore est elle-même une telle règle, elle indique les technologies d'aujourd'hui qui ne seront pas suffisantes demain. Elle organise donc la prise de conscience partagée de l'obsolescence et pousse à la rupture. Une seconde règle de ce type s'exprime par la formule "we are not picking winner or looser ». L'ITRS ne sélectionne pas, ne décide pas. Son ambition est de faire valoir et de susciter l'expression d'alternatives aussi nombreuses que possible. Elle les classe en fonction de la distance aux solutions connues dans les roadmap en fonction de la performance des connaissances manquantes et des efforts de conception nécessaires. En enrichissant l'écologie des concepts imaginables, elle déplace la frontière de l'inconnu imaginable et indique les nouvelles voies de rupture à explorer par les uns ou les autres.

Ces deux exemples nous donnent quelques indications précieuses sur le fonctionnement des " collèges de l'inconnu ", sur les conditions et les difficultés rencontrées en pratique. Les collèges de l'inconnu s'efforcent de "faire venir " tout le monde. Ou plus précisément de mobiliser les «bons concepteurs " et d'éviter les comportements opportunistes ou les phénomènes d'engouement sources de bulles spéculatives. Les « bons concepteurs » sont ceux qui voient le potentiel d'un nouveau concept inconnu et qui mesurent l'effort de conception 
nécessaire, qui y voient une occasion de mobiliser les compétences qu'ils ont déjà mais surtout de les renouveler et de les étendre. Des méthodes de gestion originales et particulièrement exigeantes sont indispensables pour gérer des formes de cohésions très particulières (les acteurs doivent coopérer alors que les intérêts ne sont pas encore connus). Les collèges de l'inconnu apparaissent aussi comme des espaces privilégiés d'invention de ces nouvelles méthodes (par exemple les référentiels de conception, Agogué, 2012).

Les collèges de l'inconnu réussissent donc à déplacer la frontière de l'inconnu accessible. Leur fonctionnement repose sur une circulation intensive des concepts, ils s'efforcent de maintenir la dimension conceptuelle de l'inconnu et de ré-ouvrir régulièrement l'exploration en désignant de nouveaux inconnus. On observe que, quand le collège de l'inconnu a déplacé la frontière de l'inconnu en organisant l'échange sur les concepts, alors l'entreprise peut reprendre la main sur ce nouvel imaginaire: celui-ci lui est devenu accessible, il est devenu " gérable " pour l'entreprise.

\title{
Retour sur l'entreprise, ses rationalisations et la dynamique des collèges de l'inconnu
}

\begin{abstract}
Pourquoi est-il plus difficile aux entreprises qu'aux collèges de l'inconnu de déplacer la frontière de l'inconnu accessible?

Lanalyse des exemples précédents nous a permis de dégager un ensemble de dimensions qui jouent un rôle critique dans la capacité à déplacer la frontière de l'imaginaire accessible. Le tableau ci-après compare sur ces différentes variables la situation des collèges de l'inconnu et de l'entreprise.
\end{abstract}




\section{PourQuoI LES COLLÈges DE L'INCONNU RÉUSSISSENT MIEUX QUE LES ENTREPRISES À EXPLORER L'INCONNU ET ÉTENDRE L'IMAGINABLE}

\begin{tabular}{|c|c|c|}
\hline & Collège de l'inconnu & Entreprise \\
\hline Intérêts & $\begin{array}{l}\text { Pas lui-même concepteur; seulement } \\
\text { expansion de l'inconnu imaginable et } \\
\text { transformation en inconnu accessible } \\
\text { à différents acteurs }\end{array}$ & $\begin{array}{l}\text { Émergence d'un nouvel inconnu } \\
\text { accessible + Appropriation partielle } \\
\text { rapide et exclusive }\end{array}$ \\
\hline $\begin{array}{l}\text { Engagement } \\
\text { des tiers }\end{array}$ & $\begin{array}{l}\text { Échange et circulation de concepts: } \\
\text { "Can you see the potential? " }\end{array}$ & Soupçonnée de visées appropriatives \\
\hline $\begin{array}{l}\text { Constitution } \\
\text { de collectifs }\end{array}$ & Fédératif/Inconnu partagé & Ségrégatif/Conflit $=$ blocage \\
\hline $\begin{array}{l}\text { Dynamique/ } \\
\text { déplacement } \\
\text { de la frontière }\end{array}$ & $\begin{array}{l}\text { Poursuivre la régénération de } \\
\text { l'inconnu et l'ouverture de nouvelles } \\
\text { voies }\end{array}$ & $\begin{array}{l}\text { Au rythme des capacités } \\
\text { d'appropriation de l'imaginable rendu } \\
\text { accessible }\end{array}$ \\
\hline $\begin{array}{l}\text { Espace } \\
\text { d'apprentissage } \\
\text { collectif }\end{array}$ & $\begin{array}{l}\text { Légitime et outillé } \\
\text { ( } \neq \text { outillage de conception habituel) }\end{array}$ & $\begin{array}{l}\text { Pas légitime à faire travailler } \\
\text { les autres chez elle }\end{array}$ \\
\hline
\end{tabular}

Ce tableau permet de formuler une hypothèse pour expliquer pourquoi les entreprises ont du mal à déplacer la frontière de l'imaginaire accessible : la tentation de l'appropriation impatiente de tout nouvel inconnu limiterait la capacité de l'entreprise à étendre l'inconnu imaginable et à déplacer la frontière de l'inconnu accessible.

\section{L'hypothèse de l'inconnu commun non appropriable}

Nous pouvons formuler l'hypothèse suivante : il existerait un « inconnu commun non appropriable ». Dans ce cas, la condition de l'extension de l'inconnu imaginable et du déplacement de la frontière de l'inconnu accessible serait précisément de redéfinir dans l'inconnu une frontière entre l'appropriable et ce qui ne l'est pas. C'est en effet avant tout l'inconnu commun non appropriable qui peut être étendu collectivement. 
Remarquons d'emblée que cette condition de non appropriabilité de l'inconnu commun ne constitue pas un obstacle pour les collèges de l'inconnu. De par leur position, ils ne sont pas directement concernés par l'appropriation des nouveaux inconnus, leur raison d'être est bien plutôt de fabriquer du commun et de favoriser un renouvellement régulier de l'inconnu.

Pour préciser cette notion d'inconnu commun non appropriable, un exemple nous vient de la science. Lorsque Dmitri Mendeleïev élabore son tableau périodique des éléments en fonction de leur masse atomique, de nombreuses cases restent vides. Elles désignent de nouveaux inconnus communs, des éléments rendus imaginables : grâce au tableau, on en connaissait certaines propriétés (par exemple des éléments analogues à l'aluminium et au silicium devaient avoir une masse atomique entre 65 et 75), mais les éléments en question étaient encore inconnus. Les nouvelles catégories d'inconnus introduites par de nouveaux langages ou méthodes ou les grandes voies conceptuelles présentées dans les référentiels en sont d'autres exemples.

\section{Communaliser les intérêts : une condition pour que l'entreprise puisse} aussi régénérer son inconnu accessible

On l'a vu, la tentation d'une appropriation prématurée de l'inconnu est l'obstacle majeur pour que l'entreprise puisse, comme les collèges de l'inconnu, étendre l'imaginaire et déplacer la frontière de l'inconnu accessible. Elle doit restaurer une frontière entre un nouvel inconnu appropriable et un nouvel inconnu commun non appropriable. En même temps que se déplace la frontière de l'inconnu accessible (qui est appropriable), doit se déplacer le domaine de l'imaginaire, celui de l'inconnu commun non appropriable. La firme ne peut s'engager dans cette voie avec d'autres sans inventer de nouveaux dispositifs communalisant les intérêts.

De tels dispositifs existent déjà pour l'inconnu accessible : c'est précisément l'objectif des secteurs et des filières industrielles. La certification aérienne ou l'Agence de veille sanitaire (pour les nouveaux médicaments améliorant la santé collective) permettent de gérer des inconnus stabilisés dans des collèges du connu. Ce n'est sans doute pas un hasard si dans le contexte contemporain, ces grands acteurs sont 
souvent confrontés à des crises sévères : celles-ci tiennent tant à l'appropriabilité tous azimuts qu’à la difficulté à faire émerger de nouveaux inconnus communs.

\section{Perspectives}

Sans dispositif pour définir et gérer la frontière entre inconnu commun et inconnu appropriable, l'extension de l'inconnu accessible restera problématique pour les entreprises. On peut donc prédire la multiplication des "collèges de l'inconnu ", mais aussi de leurs probables pathologies avec soit la multiplication d'associations se revendiquant trop rapidement de bien communs, soit avec l'apparition de tragédies des inconnus communs ${ }^{4}$. Un acteur avec une visée appropriatrice prématurée pourrait en effet " tuer " l'inconnu commun en étant fixé et en suscitant des fixations chez les autres acteurs. L'État, s'il intervenait, risquerait lui aussi de provoquer de telles fixations, à moins qu'il ne se dote de capacités pour animer ces collèges de l'inconnu.

Nous pouvons nous attendre à l'invention de nouvelles formes de l'action collectives, plus instruites et moins appropriatrices, qui se donneront comme mission l'exploration de l'inconnu et pas seulement l'apprentissage.

Les collèges de l'inconnu, s'ils se développent et se multiplient, ne manqueront pas de soulever la question de leur rationalisation. La firme, comme espace d'apprentissage particulièrement efficace pourrait alors être un candidat sérieux de cette rationalisation à condition d'inventer des dispositifs capables de communaliser les intérêts. Mais au final, il est vraisemblable qu'entreprises et "collèges de l'inconnu " constituent ensemble un dualisme indispensable au capitalisme de l'innovation intensive. 


\section{BIBLIOGRAPHIE}

Agogué M., 2012, "Modéliser l'effet des biais cognitifs sur les dynamiques industrielles : innovation orpheline et architecte de l'inconnu ", Sciences de gestion, MINES ParisTech, 253.

Arrow K. J., et Debreu G., 1954, "Existence of an equilibrium for a competitive economy", Econometrica, 22, (3), pp. 165-290.

Crane D.,1972, Invisible colleges. Diffusion of Knowledge in Scientific Communities, The University of Chicago Press.

Dosi G. et al., 2006, "Information, appropriability, and the generation of innovative knowledge four decades after Arrow and Nelson: an introduction", Industrial and Corporate Change 15(6): 891-901.

Droste M., 2002, Bauhaus 1919-1933, Köln, Taschen.

Hatchuel A., 1994, «F. Taylor : une lecture épistémologique. L'expert, le théoricien, le doctrinaire ", in Bouilloud P. et LéCuyer B. P., L'Invention de la gestion, L'Harmattan.

Heymann M., 2005, "Kunst" und Wissenchsaft in der Technik des 20. Jahrhunderts. Zur Geschichte der Konstruktionswissenschaft, Zürich, Chronos Verlag.

KöNIG W., 1999, Künstler und Strichezieher. Konstruktions und Technikkulturen im deutschen, britischen, amerikanischen und französischen Maschinenbau zwischen 1850 und 1930, Suhrkamp Verlag, Frankfurt am Main.

Le Masson P. et Weil B., 2008, «La domestication de l'innovation par les entreprises industrielles : l'invention des bureaux d'études ", Les Nouveaux Régimes de la conception. Hatchuel A. et Weil B., Vuibert-FNEGE, pp. 53-69.

Le Masson P., Weil B. \& Hatchuel A., 2010, Strategic Management of Design and Innovation. Cambridge University Press.

Le Masson P., Barbier M. et al., 2012, "The sustainable fibres of generative expectation management: The 'building with hemp' case study”. System Innovations, Knowledge Regimes, and Design Practices towards Transitions for Sustainable Agriculture, BARBIER M. and Elzen B., INRA Éditions, pp. 226-251.

Le Masson P., Weil B. et al., 2012, "Why aren't they locked in waiting games? Unlocking rules and the ecology of concepts in the semiconductor industry", Technology Analysis \& Strategic Management 24(6): 617-630.

Nelson D., 1980, Frederick W. Taylor and the Rise of Scientific Management, University of Wisconsin Press.

Nelson R., 1992, "The Roles of Firms in Technical Advance: A Perspective from Evolutionary Theory", Technology and Enterprise in a Historical Perspective, Dosi G., Giannetti R., et Toninelli P. A. (eds.), Clarendon Press, Oxford, pp.164-184.

Osтrom E., 1990, Governing the commons: The evolution of institutions for collective action, Cambridge University Press.

Schwartz F. J., 1996, The Werkbund, Design Theory and Mass Culture before the First World War. New Haven and London, Yale University Press. Vérin, H. (1993). 
VÉRIN H., 1993, La Gloire des ingénieurs, l'intelligence technique du XVI ${ }^{e}$ au XVIII ${ }^{e}$ siècle, Albin Michel.

Ward T. B., et al., 1999, "Creative Cognition”, in Sternberg R.J., Handbook of Creativity, Cambridge University Press, pp. 189-212.

Weil B., Le Masson P. et al., 2011, "Les collèges de l'inconnu : les régimes de conception de la nouvelle puissance industrielle ", Colloque final ANR programme Entreprise, Projet RITE (R\&D, Innovation et Transformation des Entreprises). 